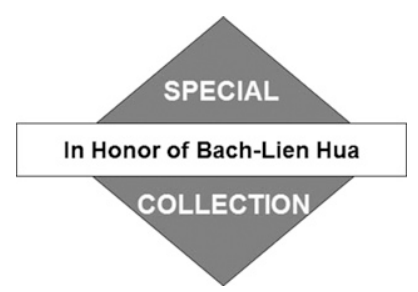

\title{
Observations of Layering under a Warm-Core Ring in the Gulf of Mexico
}

\author{
Thomas Meunier, Enric Pallàs Sanz, Miguel Tenreiro, and José OchoA \\ Physical Oceanography Department, Centro de Investigación Científica y de Educación Superior de Ensenada, \\ Ensenada, Baja California, Mexico \\ ANGEL RUIZ ANGULO \\ Icelandic Meteorological Office, Reykjavík, Iceland \\ CHRISTIAN BUCKINGHAM \\ Laboratoire d'Océanographie Physique et Spatiale, Institut Universitaire Européen de la Mer, Plouzané, France
}

(Manuscript received 28 June 2018, in final form 3 June 2019)

\begin{abstract}
Two glider transects in the Gulf of Mexico reveal fine-vertical-scale thermohaline structures within a Loop Current eddy (LCE). Partially compensating temperature and salinity anomalies are shown to organize as thin layers below the eddy and near its edges. The anomalies have vertical scales ranging from 2 to $60 \mathrm{~m}$ and extend laterally over distances up to $120 \mathrm{~km}$. These structures are evident in synthetic acoustic reflectivity derived from the glider data and are reminiscent of the intense layering observed in seismic imagery around meddies, Agulhas rings, and warm-core Kuroshio rings. The observed layers are aligned with the geostrophic streamfunction rather than isopycnals and develop preferentially in zones of intense vertical shear. These observations suggest that tracer stirring by the eddy's vertically sheared azimuthal flow might be an important process for their generation. In an attempt to rationalize this process, high-resolution quasigeostrophic simulations were performed using an idealized anticyclonic ring for the initial conditions. As the vortex destabilizes, layering rapidly develops in the model, resulting in structures similar to those found in the observation data. Passive tracer experiments also suggest that the layers form through differential advection of the tracer field by the vertically sheared flow associated with the LCE.
\end{abstract}

\section{Introduction}

Loop Current eddies (LCEs) are large (up to $200 \mathrm{~km}$ in radius) and energetic anticyclonic rings that detach sporadically from the Loop Current (e.g., Austin 1955; Leipper 1970; Leben 2005; Xu et al. 2013, among others), carrying Subtropical Underwater (SUW) as they drift westward across the Gulf of Mexico (GoM) (Cooper et al. 1990; Forristall et al. 1992). The SUW's properties sharply contrast with the Gulf Common Water (GCW) and are evident as a warm and salty (i.e., having increased spice) anomaly between the 24.5 and $26.5 \mathrm{~kg} \mathrm{~m}^{-3}$ isopycnals (Fig. 1). Since the Yucatan Strait is the GoM's only entrance for subsurface water, the thermohaline

\footnotetext{
Corresponding author: Thomas Meunier, meunier@cicese.mx
}

anomalies found in LCE's cores have a large impact on the GCW characteristics (Vidal et al. 1992; Elliott 1982; Meunier et al. 2018b). GCW is believed to be transformed SUW, through surface heat fluxes, river discharge, evaporation, precipitation, and diapycnal mixing (Hamilton et al. 2018). Of particular interest are the processes contributing to the mixing of LCE's considerable heat and salt content.

Mature LCEs are subject to strong topographic interactions, including splitting, as they reach the western GoM's platform, also referred to as the eddy graveyard (Biggs and Müller-Karger 1994), and the importance of topographic effects in the spindown of LCEs and the diffusion of their tracers is supported by observations of increased diapycnal mixing over the GoM's sloping boundaries (Ledwell et al. 2016; Dickinson et al. 2017). 


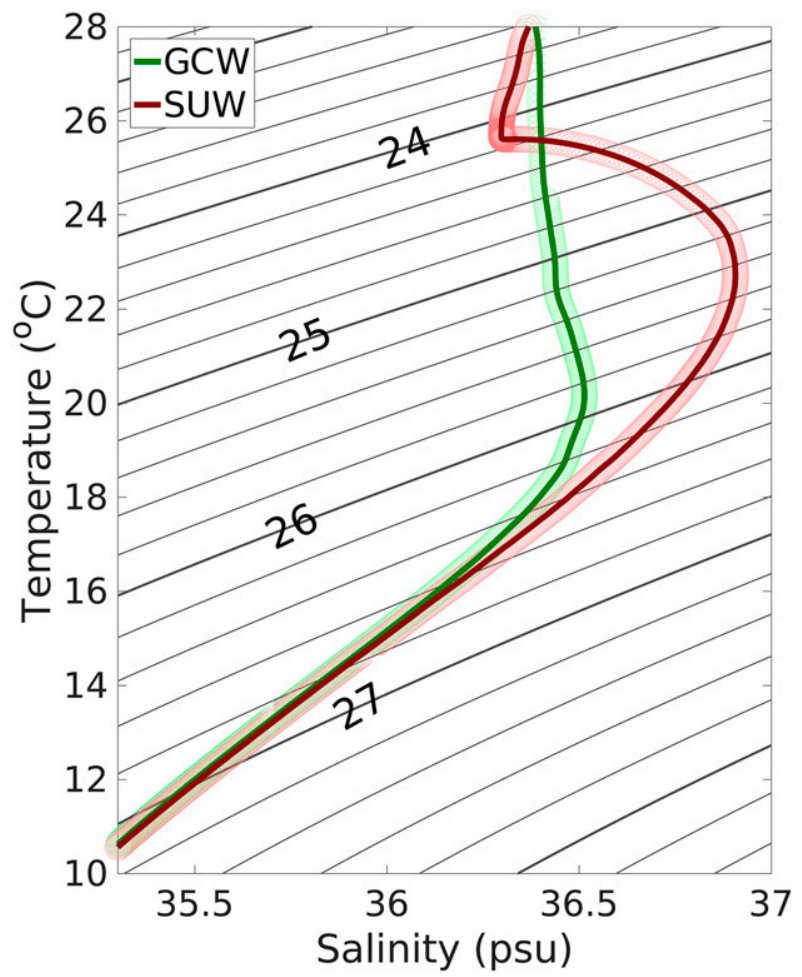

FIG. 1. A $T-S$ diagram of the two main water masses found in the subsurface in the Gulf of Mexico: the Gulf Common Water (GCW; green line), and the Subtropical Underwater (SUW; red line). The mean SUW profile was computed using glider data within the Loop Current eddy, and the mean GCW profile was computed using ARGO profiles far away from the Loop Current eddy.

However, Lipphardt et al. (2008) showed, using surface drifters and numerical modeling, that some LCEs experience intense erosion before they reach the eddy graveyard. LCEs drift several months across the GoM before reaching any topographic obstacle, and erosion of their salinity maximum was observed to start during this drift (Portela et al. 2018).

Seismic reflection images of the northern GoM (Dickinson et al. 2017) showed the presence of anomalous acoustic reflectors near the edges of, and below, the northern edge of an LCE. They are organized as vertically stacked layers with large lateral extent (up to $100 \mathrm{~km}$ ). Similar patterns were observed in seismic imagery along the edges of Agulhas rings (Rice et al. 2013) and Kuroshio warm-core rings (Yamashita et al. 2011). Conductivity-temperature-depth (CTD) and microstructure observations (Ruddick and Bennett 1985; Schmitt et al. 1986) also show similar patterns around Gulf Stream rings. These layered patterns are also reminiscent of the structures observed to form above, below, and near the edges of, Mediterranean (Sea) water eddies, or meddies (Armi et al. 1989; Ruddick and Hebert 1988; Tokos and Rossby 1991).
Joint CTD and microstructure measurements in meddies associated the layering with elevated vertical mixing (Armi et al. 1989; Ruddick and Hebert 1988). In particular, the long-term experiment of Armi et al. (1989) clearly identified erosion of a meddy, from the edges toward the center, where layering was pronounced. Similarly, both Ruddick and Bennett (1985) and Schmitt et al. (1986) found enhanced mixing near the edges of Gulf Stream rings.

Recently, Meunier et al. (2015) showed that layering around meddies resulted from a vertical tracer variance cascade driven by the vertical shear of the mean azimuthal flow. The elevated vertical mixing associated with layering could thus be related to the production of small-scale thermohaline anomalies down to the dissipative scale by a vertically sheared mesoscale flow, as previously suggested by Haynes (2001).

Although Dickinson et al. (2017) showed that diapycnal mixing was about two orders of magnitudes smaller under an LCE in deep water than in the direct vicinity of the continental slope, it might still be much larger in the presence of an LCE than in normal interior GoM conditions, far from any topographic boundary. Studying the thermohaline structure and physical origin of layering below LCEs is thus worthwhile, as the latter may constitute a route toward SUW mixing in the interior GoM. By contributing to the erosion of the temperature and salinity gradients at the edges of LCEs, layering could contribute to decrease the magnitude of the radial density gradient, and therefore play a role in the eddy's spindown by decreasing the geostrophically balanced azimuthal velocity.

In this paper, we present synoptic glider observations of the finescale thermohaline structure of layered patterns in LCE Poseidon (Meunier et al. 2018b) and use high-resolution numerical experiments to investigate the physical processes that drive their formation. Details of the glider survey and processing steps are given in section 2a. The quasigeostrophic (QG) and passive tracer advection models used in the process study are described in section $2 \mathrm{~b}$. The in situ observations are presented in section 3 , and results of the numerical experiments are described in section 4 . A summary and discussion of results are provided in section 5 .

\section{Data and methods}

\section{a. The glider data}

Measurements were made in the GoM between 5 August and 15 November 2016, using a Kongsberg 

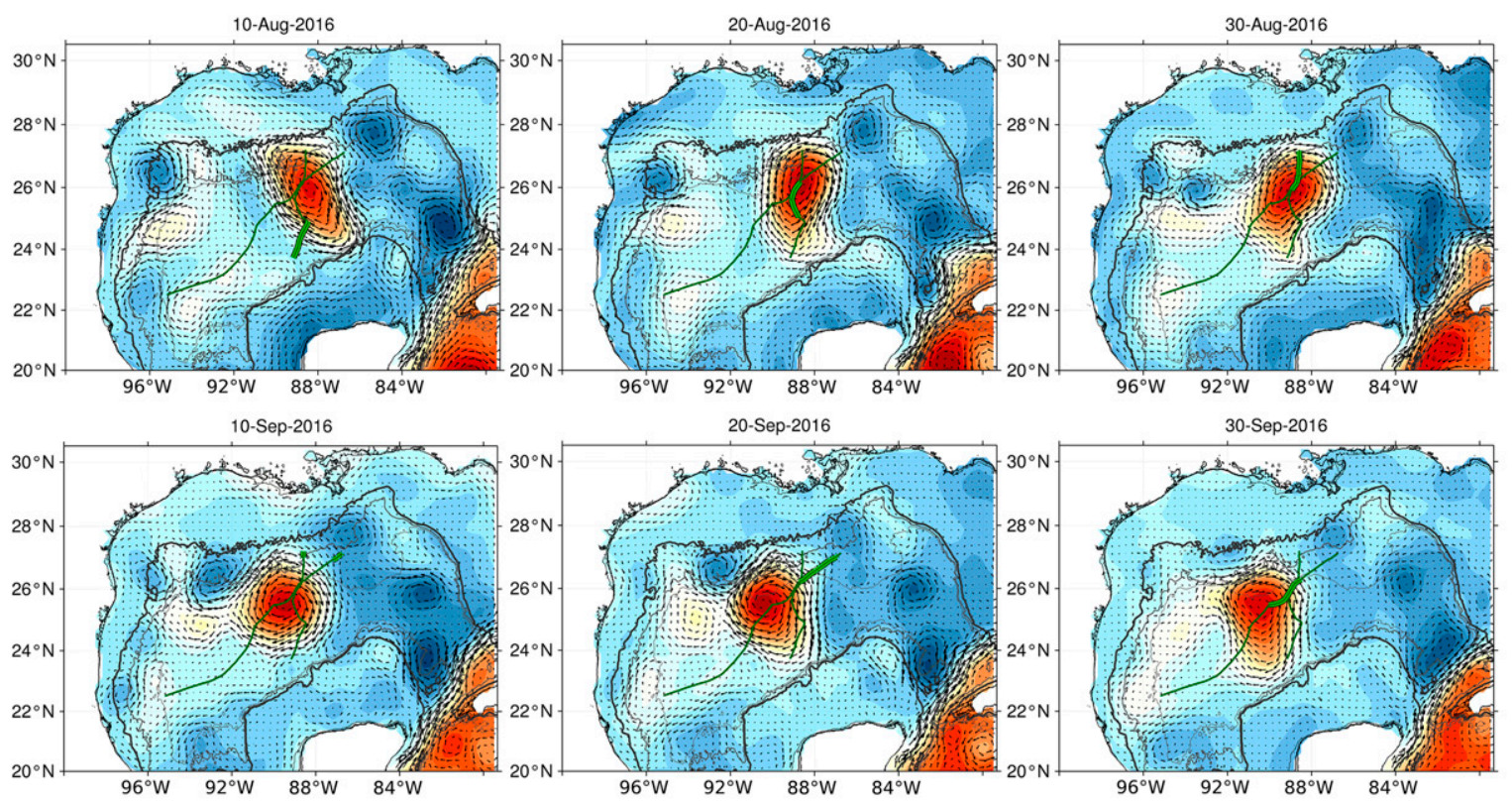

0.8
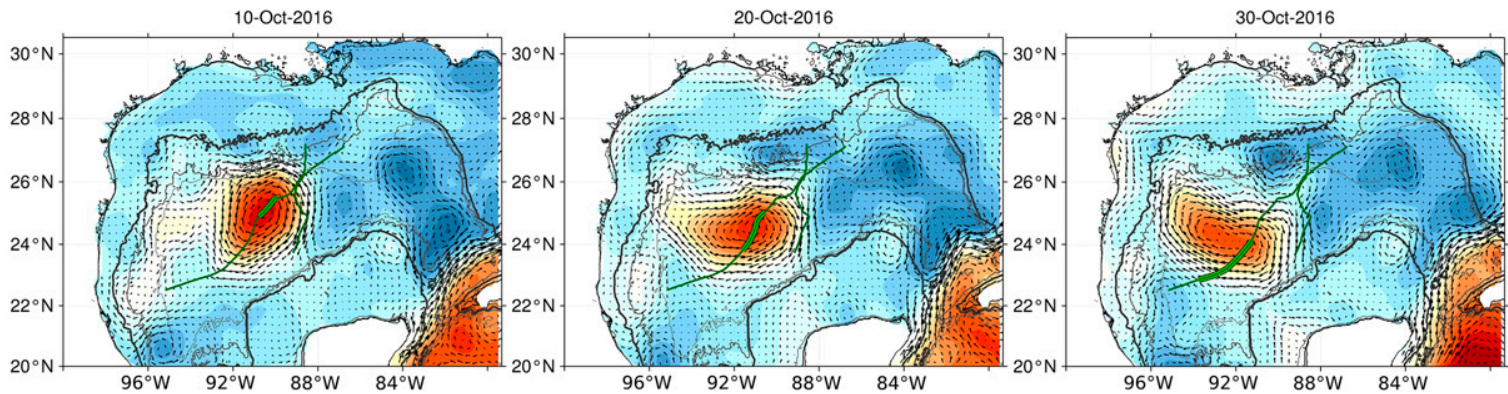

FIG. 2. Snapshots of absolute dynamic topography (ADT) from 10 Aug to 30 Oct. The geostrophic surface velocity field is indicated as black arrows. The glider's track is plotted as a dark-green line. The thick green line represents the distance traveled between 5 days before and 5 days after the ADT snapshot's date. The 2000- and 3000-m isobaths are plotted as thin gray contours, and the 1000-m isobath is represented by a thick gray contour. Section 1 was sampled from 10 Aug to 10 Sep 2016, and section 2 was sampled from 10 Sep to 5 Nov 2016.

Maritime Seaglider autonomous underwater vehicle. The glider crossed LCE Poseidon through its center twice (Fig. 2): from south to north between 10 August and 5 September 2016 (section 1) and from northeast to southwest between 10 September and 5 November 2016 (section 2).

It was equipped with a Seabird CT-sail CTD probe. The glider's average horizontal and vertical velocities were both $0.15 \mathrm{~m} \mathrm{~s}^{-1}$, and the CTD sampling rate was $0.15 \mathrm{~Hz}$, resulting in effective (mean) horizontal and vertical resolutions of $2 \mathrm{~km}$ and $2 \mathrm{~m}$, respectively. The conductivity data were corrected using an algorithm described by Garau et al. (2011) to remove thermal lag effects in zones of intense temperature gradients (Lueck 1990). A detailed description of the survey and the dataprocessing methods is given in Meunier et al. (2018b).

In an effort to better understand finescale processes, temperature and salinity profiles were high-pass filtered using a second-order Butterworth filter with a cutoff frequency of $80 \mathrm{~m}$ (Meunier et al. 2015). Conceptually, the high-pass-filtered variables can be thought of as the difference between the observed field and that of a low-pass-filtered LCE. For this reason, we hereinafter refer to high-pass-filtered variables as anomalies.

To allow comparison with recent seismic observations of layering around submesoscale interior vortices (Biescas et al. 2008; Pinheiro et al. 2010; Ménesguen et al. 2012) and in the vicinity of warm-core rings (Yamashita et al. 2011; Rice et al. 2013; Dickinson et al. 2017), synthetic acoustic reflectivity $R$ was computed. The latter is equal to the vertical derivative of acoustic impedance $I$, which is the product of sound speed $c_{s}$ and density $\rho$ (Sallarès et al. 2009):

$$
I=\rho c_{s} \text { and }
$$




$$
R=\partial_{z} I
$$

Small-scale vertical temperature gradients were shown to account for $80 \%$ of reflectivity variations in the ocean (Sallarès et al. 2009).

Because double diffusive processes were previously associated with the presence of layering around vortex lenses (Ruddick and Hebert 1988; Ruddick 1992), the Turner angle (Turner 1980; Ruddick 1983), which characterizes the diffusive stability of a water column, was computed. The Turner angle Tu, expressed in degrees, is defined as

$$
\mathrm{Tu}=\arctan \left(\alpha \partial_{z} T-\beta \partial_{z} S, \alpha \partial_{z} T+\beta \partial_{z} S\right),
$$

where $T$ is temperature; $S$ is salinity; $\alpha$ and $\beta$ are the thermal expansion and the saline contraction coefficients, respectively; and $\arctan (y, x)$ is the quadrant-preserving implementation of the arctangent function. One observes that the water column is diffusively unstable for $-90^{\circ}<\mathrm{Tu}<-45^{\circ}$, stable for $-45^{\circ}<\mathrm{Tu}<45^{\circ}$, and salt-finger unstable for $45^{\circ}<\mathrm{Tu}<90^{\circ}$ and is otherwise is statically unstable (Ruddick 1983).

Geostrophic velocity was computed from the in situ density and referenced using the depth-averaged currents, computed as the difference between 1) the observed drift of the glider during each dive and 2) that predicted by the inboard flight model (Rudnick et al. 2015).

Geostrophic streamfunction was computed from the in situ density anomaly $\rho^{\prime}$. The latter is defined as the difference between a measured density profile and an average GoM profile computed using 39 Argo profiles outside the LCE between 1 April and 1 November 2016 (Meunier et al. 2018b). The geostrophic streamfunction $\psi_{g}$ is defined as

$$
\begin{aligned}
\psi_{g} & =\int_{-H}^{0} \frac{g}{f \rho_{o}} \rho^{\prime} d z, \\
\rho^{\prime} & =\rho(s, z)-\bar{\rho}(z),
\end{aligned}
$$

where $s$ is the distance along the glider path, $g$ is the gravity acceleration, $f$ is the Coriolis parameter, and $H$ is the greatest sampled depth, assumed to correspond to the level of no motion. In the model simulations below, $s=r$ becomes the radial coordinate from the eddy center.

\section{b. Numerical models}

\section{1) The quasigeostrophic Model}

Here, we use a pseudospectral QG model (Hua and Haidvogel 1986) which was employed previously in various studies on the dynamics of layering around oceanic vortex lenses (Hua et al. 2013; Meunier et al. 2015, 2018a; Ménesguen et al. 2018). Apart from the lower computational cost when compared with performing simulations using primitive equation models, use of the QG framework offers the distinct advantage of simplifying the basic dynamical mechanisms, facilitating understanding of the physical processes at work. Since neither internal waves nor double diffusion processes exist in this model, a successful simulation of layering in the model would suggest that the leading-order mechanism is not internal waves or double diffusion. If we can successfully reproduce the observations with a simple model, we have made a much more powerful statement about what dynamics are associated with this process.

The model integrates the QG potential vorticity (PV) evolution equation:

$$
{ }_{t} q+J(\psi, q)=\nu \nabla^{6} \psi
$$

Here, $\psi$ is the streamfunction, $J(A, B)=\partial_{x} A \partial_{y} B-$ $\partial_{y} A \partial_{x} B$ is the two-dimensional Jacobian, and the $\mathrm{PV}$ is defined as follows:

$$
q=\nabla^{2} \psi+\partial_{z}\left(\frac{f^{2}}{N^{2}} \partial_{z} \psi\right),
$$

where $N$ is the Brunt-Väisälä frequency. A dissipation term has been added on the right-hand side of Eq. (6) in the form of a biharmonic operator $\nabla^{6} \psi$ to avoid numerical instability. The diffusion coefficient $\nu$ is set as small as possible to approximately conserve PV. For computational efficiency, PV inversion is performed in spectral space, and advection is performed in physical space. Additional details of the numerics are documented in Hua and Haidvogel (1986) and Hua et al. (2013). The vertical and horizontal grid steps are $d z=5.3 \mathrm{~m}$ and $d x=1000 \mathrm{~m}$. They were chosen to ensure high vertical resolution while satisfying Charney's isotropy criterion, $d x / d z \approx N / f$, which has been shown to be important for convergence of energy spectra (Ménesguen et al. 2018). The domain is $1920 \mathrm{~m}$ deep, $500 \mathrm{~km}$ wide, and double periodic. The domain size was chosen to be approximatively equal to the distance between the southern and northern GoM's continental slopes near $88^{\circ} \mathrm{W}$. Sensitivity experiments were performed ensuring that the domain was large enough to avoid interactions of the eddy with itself across the periodic boundary conditions.

The QG diagnostic density anomaly $\rho_{q}$ was also computed so as to construct a QG-equivalent reflectivity $R_{q}$ : 


$$
\begin{aligned}
& \rho_{q}=\frac{f \rho_{o}}{g} \partial_{z} \psi, \\
& R_{q}=\partial_{z} \rho_{q}^{\prime},
\end{aligned}
$$

where $\rho_{q}^{\prime}$ is the high-pass-filtered QG diagnostic density. The same Butterworth filter and cutoff frequency were used for the model outputs and the data. Note that QG reflectivity $R_{q}$ is proportional to the vortex stretching anomaly $\eta=\partial_{z}\left[\left(f^{2} / N^{2}\right) \partial_{z}(\psi-\bar{\psi})\right]$, where $\bar{\psi}$ is the basestate streamfunction, discussed by Hua et al. (2013) in their seminal paper on the dynamics and energetics of layering.

\section{2) THE TRACER ADVECTION MOdeL}

In addition to the simulations described above, we also independently performed simulations with a passive tracer model embedded in a stationary velocity field. The passive tracer advection model solves the conservation equation for a passive tracer concentration $\chi$ as follows:

$$
\partial_{t} \chi+J\left(\bar{\psi}_{s}, \chi\right)=0
$$

where $\bar{\psi}_{s}$ is the stationary streamfunction. This model is based on the tangent linear version of the Hua and Haidvogel (1986) QG model (Yen Nguyen et al. 2012; Meunier et al. 2018a). A complete description of the model can be found in Meunier et al. (2015). Below, we describe the expression that provides initial conditions for the QG model and the constant velocity field employed in the advective model.

\section{3) EDDY REPRESENTATION IN THE TWO MODELS}

In both models, the LCE was modeled using a Cauchy distribution function:

$$
\begin{aligned}
& \psi=\tilde{\psi}_{o}\left(1+d^{2}+d^{4}\right)^{-1}, \\
& d=\left[\frac{r^{2}}{L^{2}}+\frac{\left(z-z_{o}\right)^{2}}{H^{2}}\right]^{1 / 2},
\end{aligned}
$$

where $z$ is depth, $z_{o}$ is the depth of the velocity maximum, $L$ is the eddy radius, $H$ is the eddy thickness, and $\tilde{\psi}_{o}$ is a nondimensional parameter controlling the amplitude of $\psi$. To best match the observed characteristics of LCE Poseidon, the parameters were set to $H=285 \mathrm{~m}$, $L=150 \mathrm{~km}$, and $z_{o}=-80 \mathrm{~m}$. Note that in the QG model this streamfunction is used as an initial condition that then evolves with time whereas in the passive tracer experiment it remains stationary. Also note, the purpose of the numerical simulations is to contextualize the formation process of the observed layering in a simple framework. In particular, they allow us to compare the observed layering features with those formed around vortex lenses as studied by Meunier et al. (2015).

\section{In situ observations}

Poseidon is a large ( $170 \mathrm{~km}$ radius) anticyclonic eddy that detached from the Loop Current in April of 2016 and drifted southwestward through the GoM during the glider survey (Fig. 2). Its temperature distribution consists of a homogeneous warm core between depths of 50 and $250 \mathrm{~m}$, resulting in the plunging of the sub- $20^{\circ} \mathrm{C}$ isotherms (Figs. 3a,d). The salinity structure (Figs. 3b,e) shows a double core pattern, with a saline core reaching 36.95 psu, made of SUW (Wüst 1964; Hernández-Guerra and Joyce 2000) positioned between $|z|=220$ and $650 \mathrm{~m}$, and a fresher subsurface core between $|z|=30$ and $220 \mathrm{~m}$. The resulting density distribution (not shown) consists of a pycnostad between $|z|=30$ and $200 \mathrm{~m}$, confined between a surface seasonal pycnocline and the main pycnocline. The geostrophic velocity field (Figs. 3c,f) features velocity maxima exceeding $1 \mathrm{~m} \mathrm{~s}^{-1}$ below the surface, at about $150 \mathrm{~km}$ from the eddy's rotation axis. The shear is enhanced within the first $300 \mathrm{~m}$ (up to $5 \times 10^{-3} \mathrm{~s}^{-1}$ ).

High-pass-filtered temperature $T^{\prime}$, salinity $S^{\prime}$, and spice sections are shown in Fig. 4. Stacking of highwave-number anomalies of alternating signs is obvious as layered patterns in both sections: (on the $x$ axis) between -200 and $-115 \mathrm{~km}$, between -80 and $-20 \mathrm{~km}$, and between 90 and $115 \mathrm{~km}$ in section 1 and between -240 and $-120 \mathrm{~km}$ and between 180 and $205 \mathrm{~km}$ in section 2 . The salinity and temperature anomalies reach $0.07 \mathrm{psu}$ and $0.22^{\circ} \mathrm{C}$, respectively. The layers have lateral extent ranging from 30 to $120 \mathrm{~km}$.

A pair of stacked negative and positive anomaly layers marks the lower edge of the LCE's pycnostad. They extend from the surface near the edges of the eddy down to $|z|=200-\mathrm{m}$ depth, near its center. These features arise because vertical gradients are sharp in the main thermocline of the LCE, and the cutoff wavelength of the high-pass filter is too large to filter it out. Hence, they are spurious results of the filtering method and are not to be mistaken with the layering discussed above.

To assess the finescale vertical structure of the layers, vertical sections of the contributions of temperature $\left(-\sigma_{0} \alpha T^{\prime}\right)$ and salinity $\left(\sigma_{0} \beta S^{\prime}\right)$ to the potential density anomaly are shown in Fig. 5, where $\sigma_{0}$ is the mean potential density referenced to the surface. The location of the profiles is indicated by red vertical lines in Fig. 4. The vertical profiles reveal a partial compensation of the effects of temperature and salinity on density anomaly, evident as the axisymmetry of the red $\left(-\sigma_{0} \alpha T^{\prime}\right)$ and 

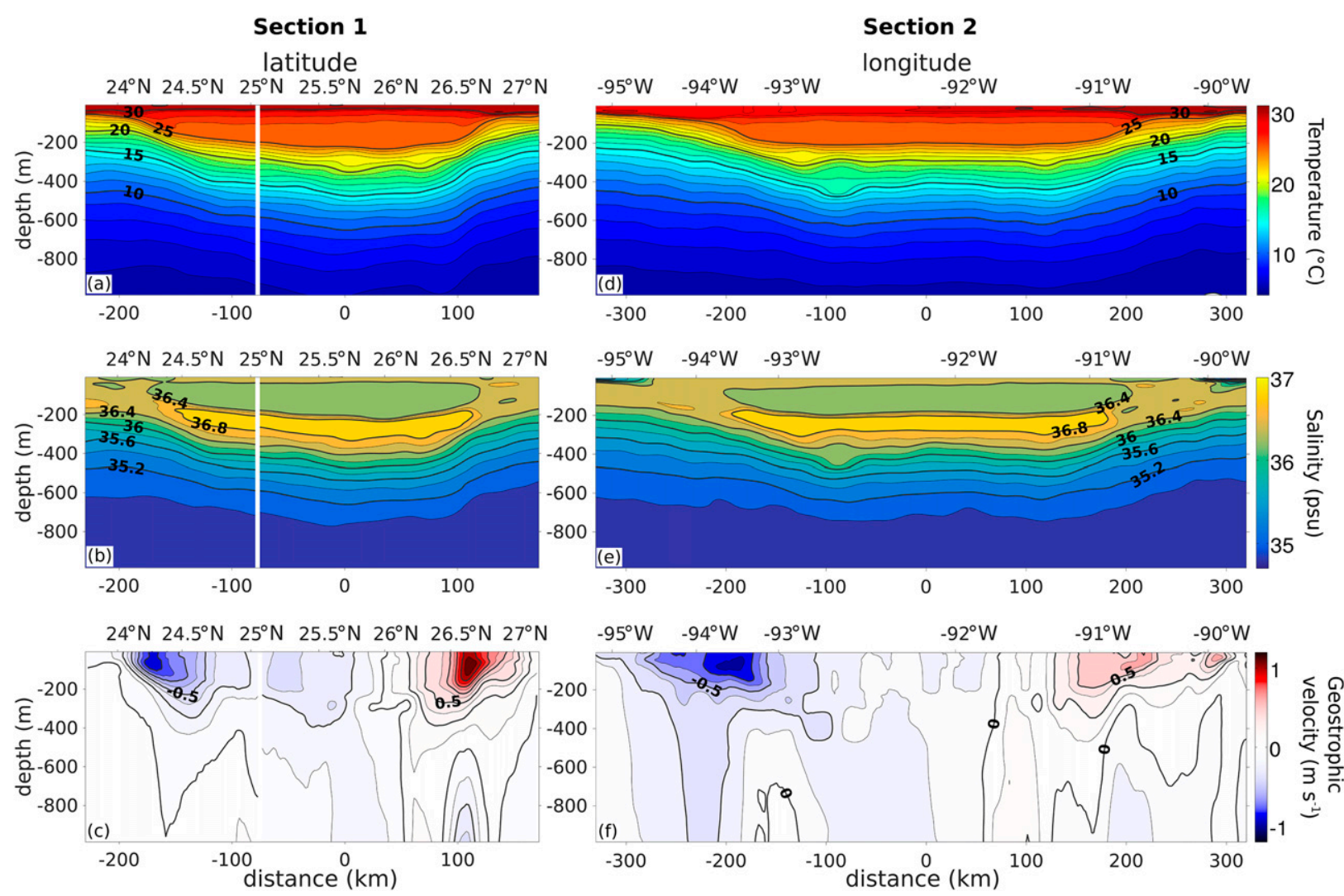

FIG. 3. The (a),(d) temperature; (b),(e) salinity; and (c),(f) geostrophic velocity from the glider transect through sections (left) 1 and (right) 2 .

blue $\left(\sigma_{0} \beta S^{\prime}\right)$ curves across the zero line. Oppositely, the spurious layers (evident as the uppermost anomaly in each profile) are not density compensated. Density compensation results in well-defined spice anomaly layers, whose amplitudes reach up to $0.27 \mathrm{~kg} \mathrm{~m}^{-3}$ and are one order of magnitude larger than those of density layers. The average thickness of the density-compensating layers, computed as the vertical distance between two different zero crossings of spice anomalies, is $20.5 \mathrm{~m}$, with a standard deviation of $11.7 \mathrm{~m}$.

The small-scale vertical variations of temperature and salinity project onto the Turner angle (Figs. $6 c, f$ ). The water column is mainly salt-finger unstable between the depths of 250 and $600 \mathrm{~m}$ below the $\operatorname{LCE}\left(50^{\circ}<\mathrm{Tu}<80^{\circ}\right)$ and a convectively unstable layer $\left(-60^{\circ}<\mathrm{Tu}<-45^{\circ}\right)$ extends all across the eddy between 200 and $170 \mathrm{~m}$, at the base of the pycnostad core.

Recent seismic imaging of the water column in the Gulf of Mexico by Dickinson et al. (2017) revealed the presence of stacked reflectors with a significant lateral extent below and at the northern edge of a small LCE. Although the glider's horizontal resolution is about 200 times as coarse as that of seismic imaging, its vertical resolution is about 5 times as fine $(2$ vs $10 \mathrm{~m})$, and comparison between the phenomenon observed during the glider survey of LCE Poseidon and previous seismic surveys of Dickinson et al. (2017) is worthwhile. Figures $6 \mathrm{a}$ and $6 \mathrm{~d}$ show synthetic acoustic reflectivity computed from the glider-measured temperature and salinity for sections 1 and 2, respectively. Because the LCE's core is nearly homogeneous, it appears as a thick body of low reflectivity in the top $200 \mathrm{~m}$. Two of the three intrathermocline eddies discussed in Meunier et al. (2018c) also have a clear low-reflectivity signature, especially obvious in section 2 at -120 and $-70 \mathrm{~km}$ and $|z|=250$ and $550 \mathrm{~m}$. Below the LCE's core, at depths between $|z|=150$ and $400 \mathrm{~m}$, one observes clear vertically stacked layers of anomalous reflectivity near the lateral edges, and in the central part of section 1 . These are coincident with the temperature and salinity anomalies shown in Fig. 4. This suggests that the laterally coherent thermohaline anomalies sampled with the glider might be of the same nature as the seismic reflectors shown by Dickinson et al. (2017). In both sections, the layers seem to develop preferentially below and near the edges of the LCE. The lateral extent of the layers is greater on the southern edge (left-hand side of the panels) of the LCE (60 and $120 \mathrm{~km}$ of maximal 
Section 1
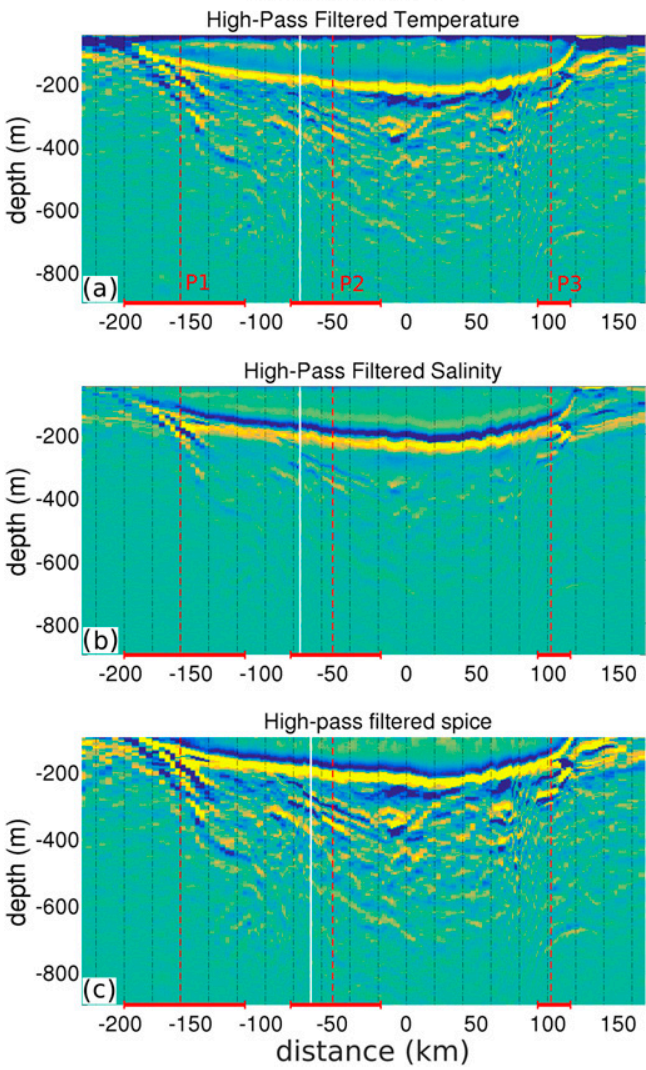

\section{MEUNIER ET AL.}

Section 2
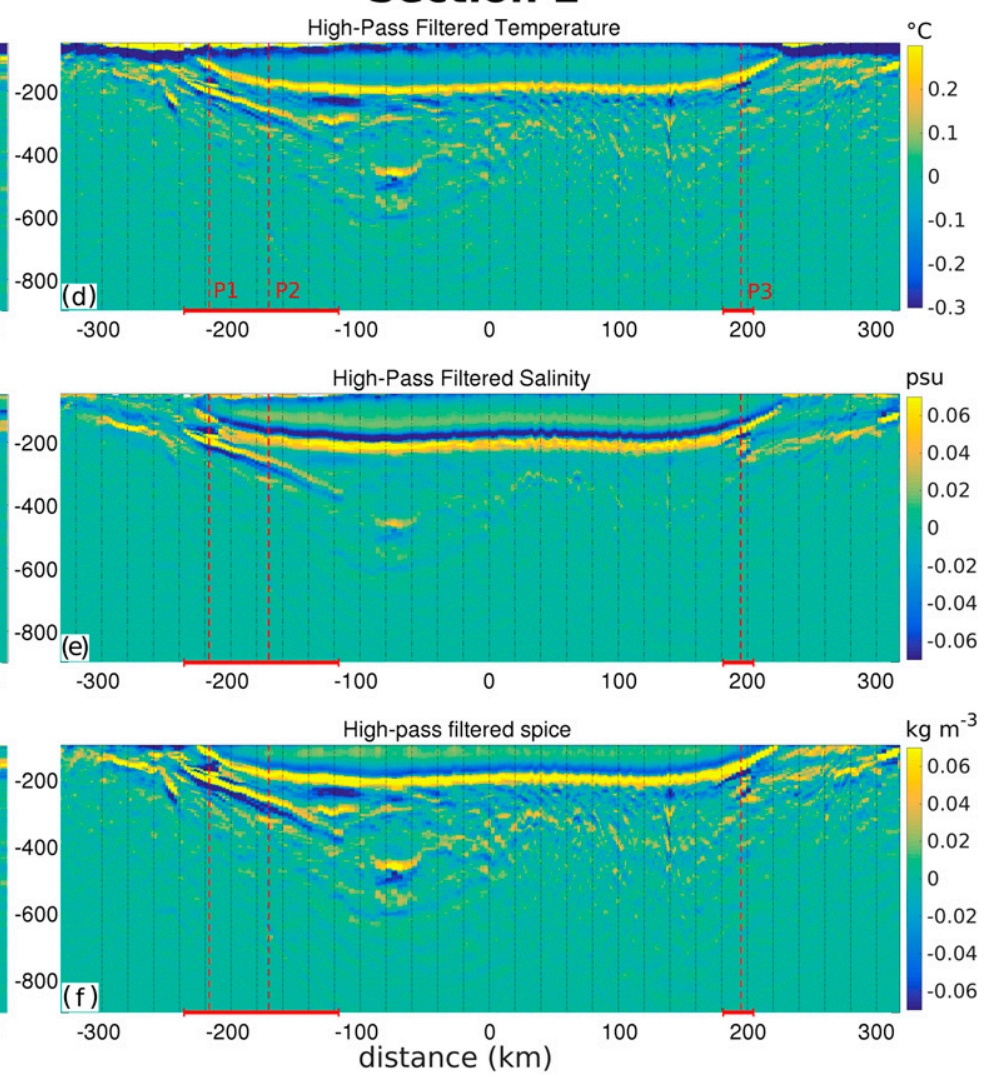

FIG. 4. High-pass-filtered (a),(d) temperature; (b),(e) salinity; and (c),(f) spice anomalies for sections (left) 1 and (right) 2 . The thick red dotted vertical lines materialize the localization of the vertical profiles of Fig. 5, below. The horizontal red lines on the $x$ axis highlight the areas where laterally coherent layering is obvious. For each section, north is on the right-hand side and south is on the left-hand side.

lateral extent in sections 1 and 2, respectively) than on the northern edge ( 30 and $20 \mathrm{~km}$ ).

In a microstructure study of a Gulf Stream ring, Schmitt et al. (1986) concluded that the action of a vertical shear on preexisting thermohaline variability could lead to the formation of intrusions at the edge of the eddy. Similar patterns found around oceanic vortex lenses were analyzed by Meunier et al. (2015), who confirmed that layering could be attributed to the stirring of a tracer (whether passive or active) by a vertically sheared flow, resulting in the alignment of the tracer gradient with the streamfunction contours.

Contours of the geostrophic vertical shear are superimposed on synthetic acoustic reflectivity for sections 1 and 2 in Figs. $6 \mathrm{~b}$ and $6 \mathrm{e}$, respectively, and geostrophic streamfunction contours are shown in Figs. 6a and 6d. The location of the layers is coincident with the zone of high vertical geostrophic shear between 50- and 250-m depth, near the edges of the eddy, in both sections. In contrast, the shear is moderate between 170 and $230 \mathrm{~km}$ in section 1 , where the layering is observed. In both sections, the reflectivity layers align with the geostrophic streamfunction, including in the central part of the LCE (Figs. 6a,d). The apparent preferential development of layering in zones of intense velocity shear, as well as the alignment of the layers with the geostrophic streamfunction, encourages a further examination of the effects of geostrophic stirring associated with an LCE-like vortex. This is performed numerically in next section.

\section{Model results}

The QG model was integrated for 100 days using the parameters described in section $2 \mathrm{~b}$. A vertical section of the streamfunction and the corresponding velocity field used to initialize the QG model and to advect the passive tracer is shown in Fig. 7.

Cross sections of PV at 50-m depth after $t=0,15$, and 30 days of model integration are shown in Figs. 8a-c, respectively, and the QG density field at the same time steps is shown in Figs. 8d-f. After 15 days of integration, 


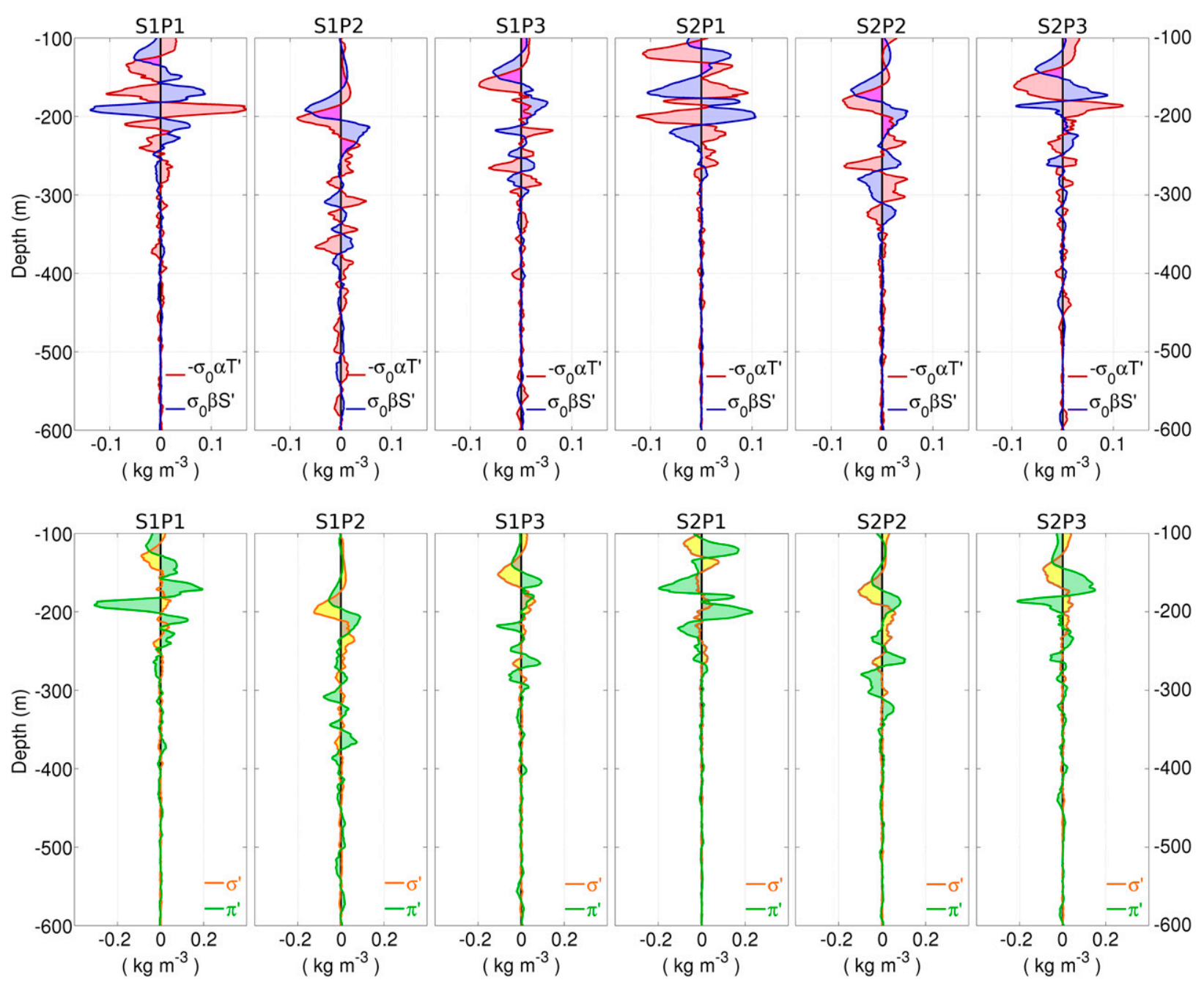

FIG. 5. Vertical profiles of (top) the contributions of temperature $\left(-\sigma_{0} \alpha T^{\prime}\right.$; red line) and salinity $\left(-\sigma_{0} \beta S^{\prime}\right.$; blue line) to the high-passfiltered density anomaly and (bottom) high-pass-filtered density ( $\sigma^{\prime}$; orange line) and spice ( $\pi^{\prime}$; green line) anomalies. Axisymmetry of $-\sigma_{0} \alpha T^{\prime}$ and $-\sigma_{0} \beta S^{\prime}$ across the zero line indicates compensating effects of the temperature and salinity anomalies.

unstable waves with azimuthal modes ranging from 2 to 6 appear to form at the outer edge of the vortex, distorting the PV contours and breaking the azimuthal symmetry. This evolution can also be seen in the density field, as density anomalies organize to a pattern matching the one seen in PV. After 30 days, azimuthal mode 2 dominates over the initially faster-growing higher modes. As a result, QG density anomalies wrap around the edge of the eddy.

The evolution of the QG reflectivity [Eq. (9)] is shown on Fig. 9. The streamfunction and vertical velocity shear are superimposed as orange and blue contours, respectively. After 15 days of integration, layering starts to form in the subsurface, near the edges of the vortex, where the vertical velocity shear is high. At $t=30$ and 60 days, the amplitude of the reflectivity anomalies increases. The layers exhibit more complex patterns as the eddy distorts because of instability, and the zones of strong vertical shear become fragmented. The presence of layering in the central part of the eddy after 30 days resembles the observations from section 1 , between 170 and $230 \mathrm{~km}$, where layering was associated with moderate shear. In the model, this happens when the amplitude of the perturbations become large (Meunier et al. 2015). At this stage, the velocity distribution shows more complex submesoscale patterns, possibly advecting previously formed layers and homogenizing them across the eddy. The layers, however, remain parallel to the streamfunction and, although they have spread laterally below the eddy center and near its edges, still have larger amplitudes immediately below the fragments of higher vertical shear.

To assess the effect of the vertically sheared velocity field on tracer distribution, the tracer advection model 

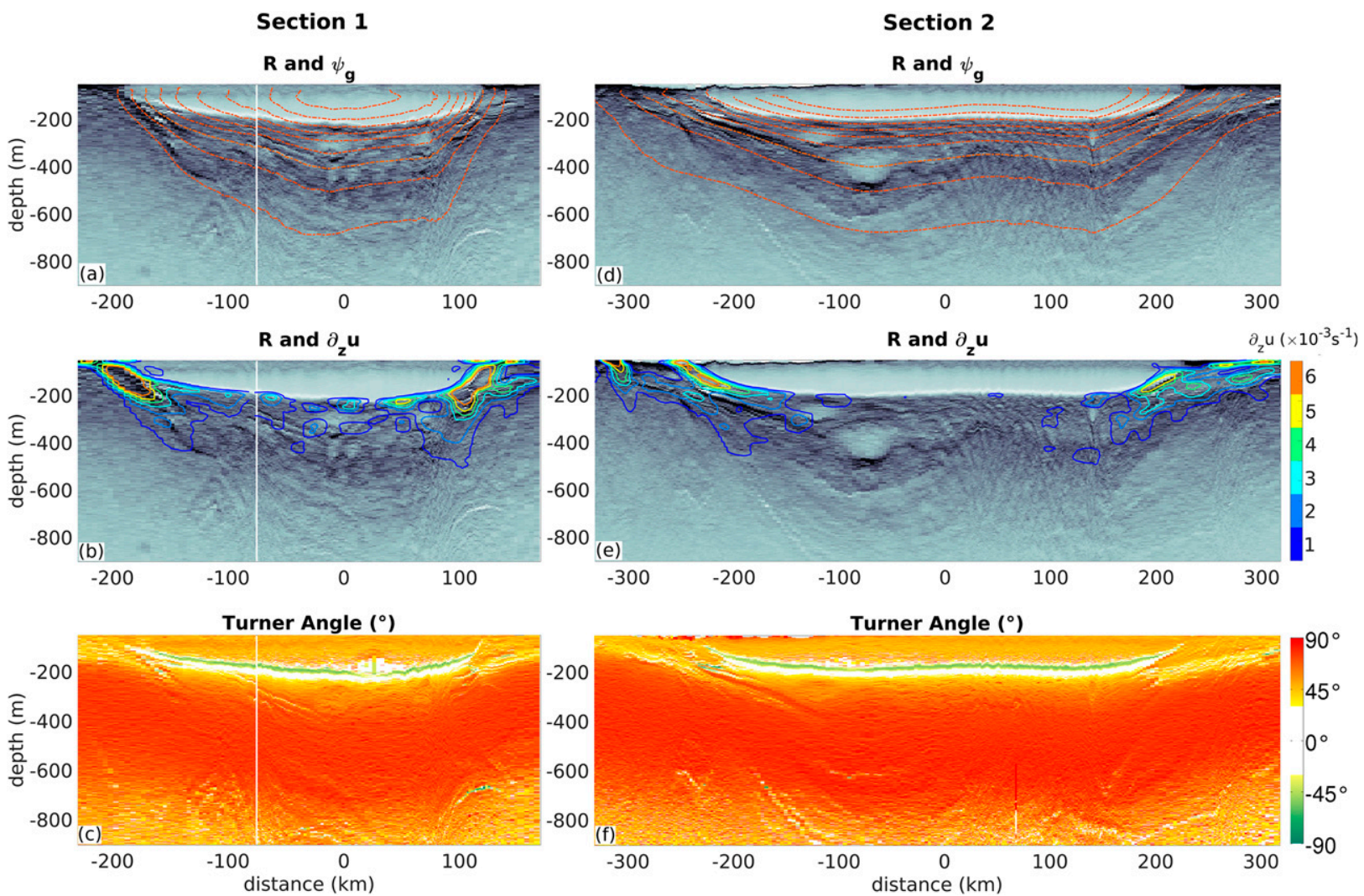

FIG. 6. (a),(d) Geostrophic streamfunction contours superimposed on vertical sections of synthetic acoustic reflectivity. (b),(e) Geostrophic velocity shear contours superimposed on vertical sections of synthetic acoustic reflectivity. (c),(f) Vertical sections of the Turner angle $\mathrm{Tu}$. A Tu $>45^{\circ}$ indicates a salt-fingering regime, $\mathrm{Tu}<-45^{\circ}$ indicates a diffusive-convection regime, and $-45^{\circ} \leq \mathrm{Tu} \leq 45^{\circ}$ indicates a stable regime. The plots are for (left) section 1 and (right) section 2; for each section, north is on the right-hand side and south is on the lefthand side.

was initialized with a horizontally random and vertically uniform tracer distribution (Figs. 10a,b) (Meunier et al. 2015). The evolution of the tracer properties after $t=$ 14 days of model integration is shown in Fig. 10. The tracer wraps around the vortex in the horizontal under the influence of the strain field (Fig. 10d) while the initially barotropic columns are tilted and deformed by the vertical shear of the azimuthal flow (Fig. 10e). Hence, the vertical component of the tracer gradient, which is initially zero, quickly grows below the eddy in the form of laterally coherent concentric stacked layers, aligning with the streamfunction where the velocity shear is high (Fig. 10f). After 14 days, some layers remain coherent over a full eddy radius. The horizontal passive tracer variance spectrum was computed and is compared with the glider-observed spice variance spectra in Fig. 11: the

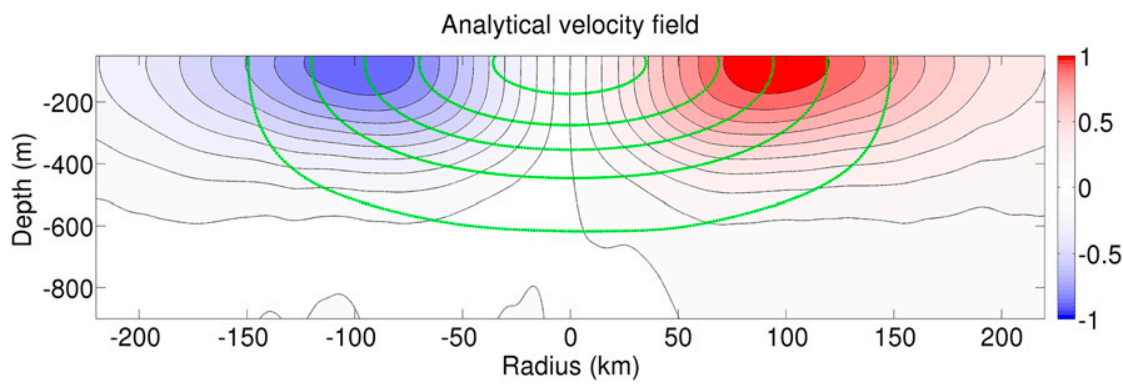

FIG. 7. Velocity field used in the QG and tracer advection model (color shading), with the Cauchy-Lorentz streamfunction plotted as green contours. 

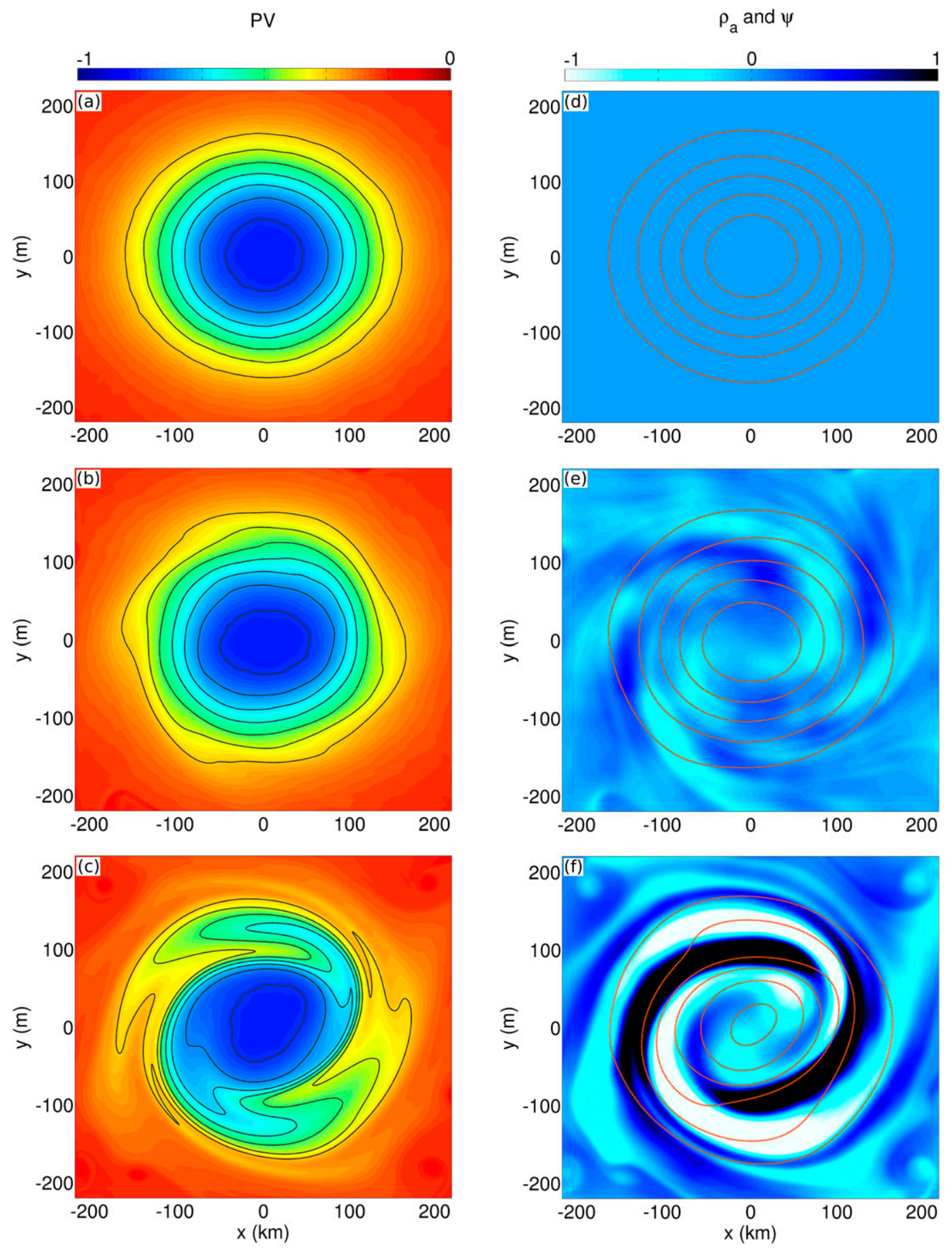

FIG. 8. Time evolution of (left) normalized PV (black contours are the PV every 0.05) and (right) normalized QG density anomaly (streamfunction superimposed as orange contours every $1000 \mathrm{~m}^{2} \mathrm{~s}^{-1}$ ) for snapshots corresponding to (a),(d) 0 ; (b),(e) 15; and (c),(f) 30 days of time integration.

observed spice's spectral slope is slightly flatter $\left(\approx k^{-2.7}\right)$ than that of the modeled passive tracer $\left(\approx k^{-3.5}\right)$, consistent with the presence of submesoscale patterns in the observed LCE.
To test the practical effect of the glider's lack of synopticity on the results presented here, the QG model outputs were resampled, simulating a glider survey (one profile every $3 \mathrm{~h}$ ), from day 15 to day 45 of the model 

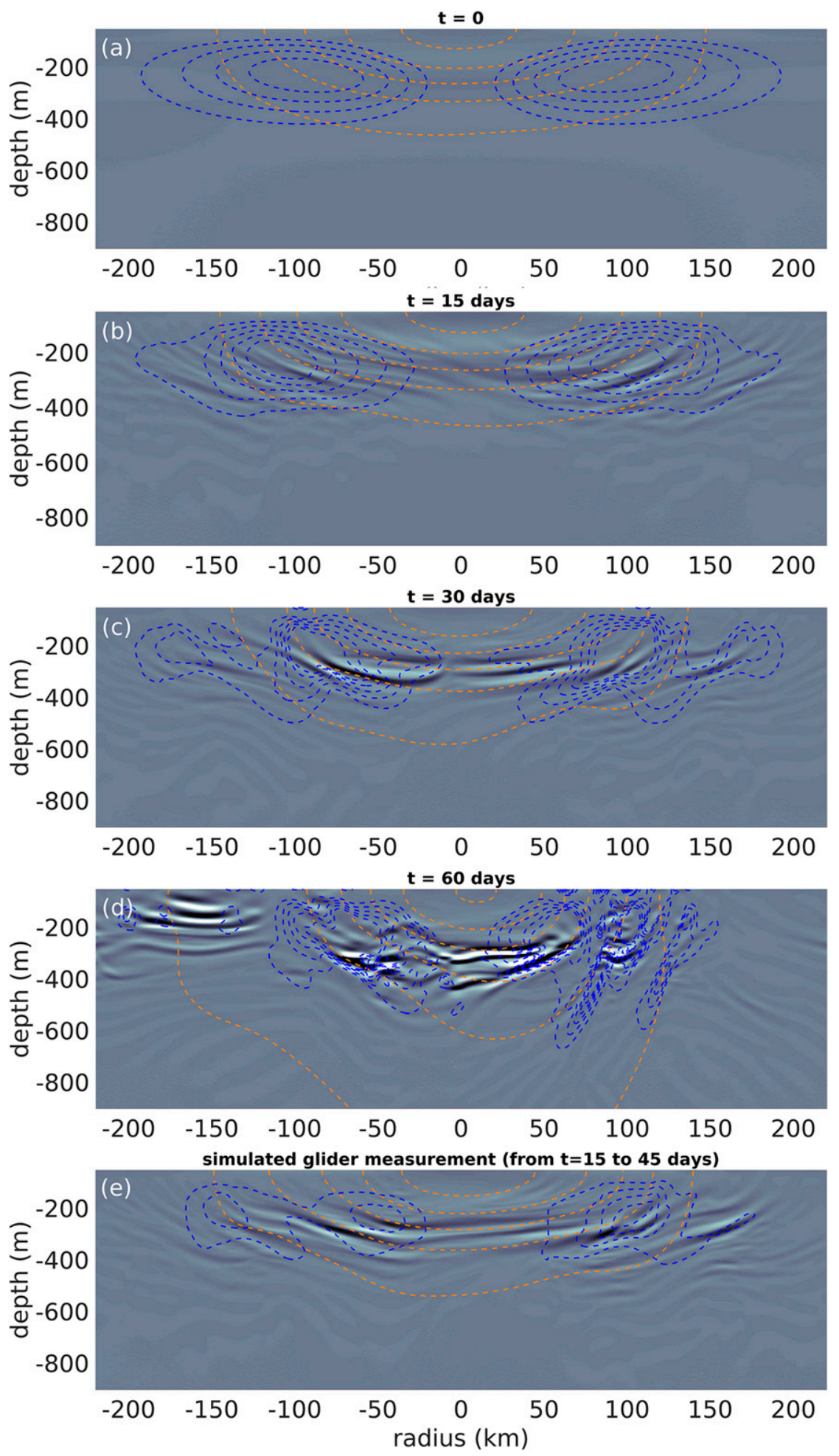

FIG. 9. QG reflectivity (grayscale image), vertical shear (blue contours), and streamfunction (orange contours) after (a) 0, (b) 15, (c) 30, and (d) 60 days of QG model run; also shown is (e) a simulation of the acquisition of the model outputs by a glider sampling a grid point every $1.5 \mathrm{~h}$. 

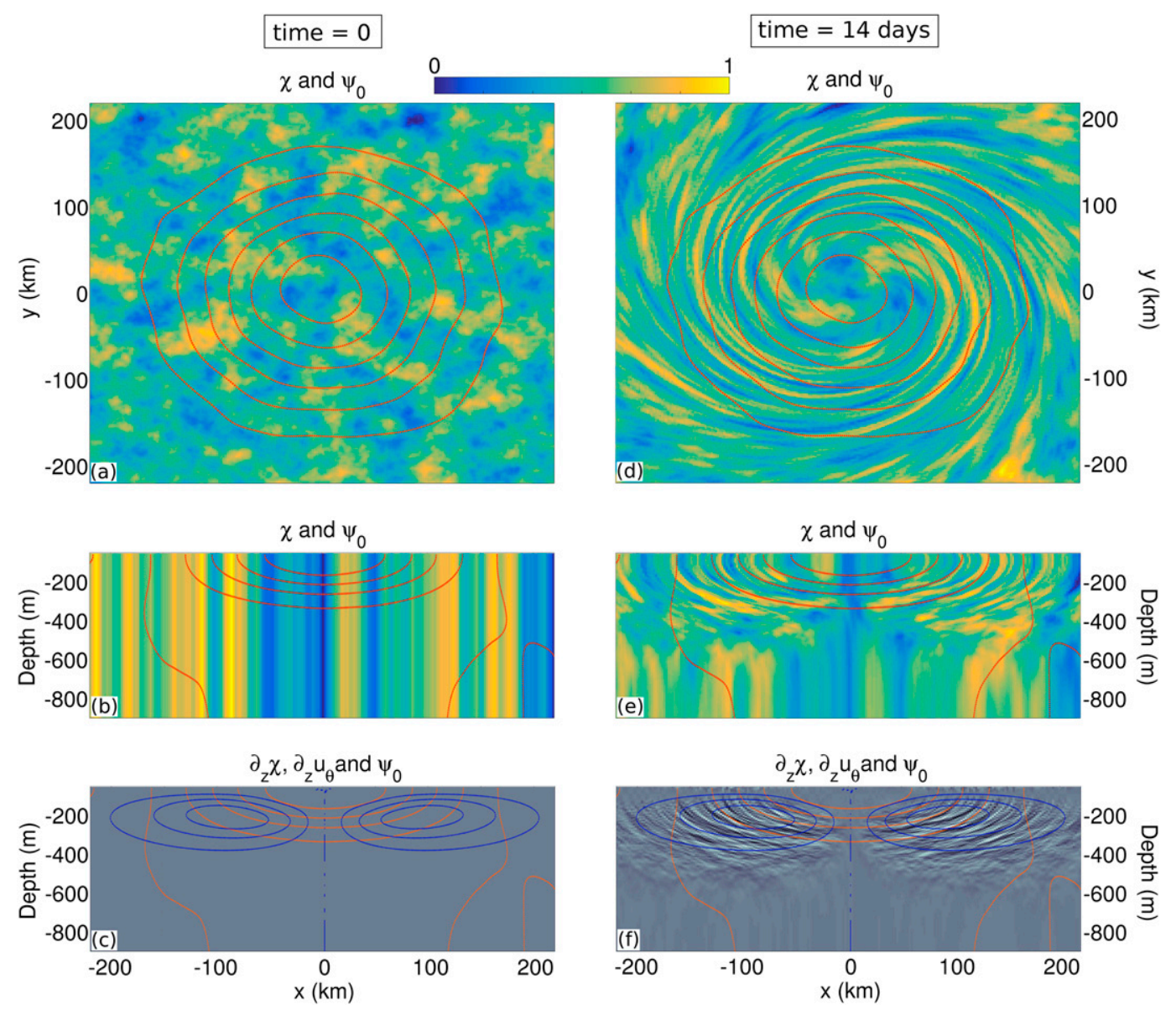

FIG. 10. Time evolution of the passive tracer in the advection model: (a) initial horizontal distribution of the tracer concentration at $50 \mathrm{~m}$, (b) initial vertical distribution of the tracer concentration at $50 \mathrm{~m}$ (color scale), and (c) initial vertical distribution of the tracer concentration's vertical gradient (color scale), with the vertical shear of azimuthal velocity plotted as blue contours. In all panels, streamfunction is plotted as orange contours. (d)-(f) As in (a)-(c), but after 14 days of model integration.

run. Results are shown in Fig. 9e. The similarity between the glider-sampled model output and the snapshot taken at the median time $t=30$ days (Fig. 9c) is striking. The lateral extent of the layers, which is expected to be affected by the lack of synopticity, is well represented, with some layers having a lateral extent of nearly $100 \mathrm{~km}$, similar to that observed in the snapshots. This suggests that, though the glider sections do not represent a single snapshot in time, they reasonably capture the thermohaline layering on the relevant horizontal and vertical length scales. This gives us good confidence in the use of our glider transects for this particular study.

\section{Discussion and conclusions}

The results presented above have combined fineresolution in situ observations and numerical modeling with the overarching aim of bringing insight to the nature and origin of layering underneath mesoscale eddies. Temperature and salinity across the layers were shown to compensate in their contribution to density anomaly, resulting in large spice anomalies and moderate density anomalies near the edges of the eddy (one order of magnitude smaller), typical of intrusions or interleaving at the boundary of two water masses with contrasting thermohaline properties (Ruddick and Richards 2003). Strong spice anomalies associated with weak density anomalies have been shown to behave like a passive tracer (Flament 2002), suggesting the possible role of stirring in the formation of the observed layers (Song et al. 2011; Pinheiro et al. 2010). Evidences for preferential development of layering in zones of high vertical shear below the ring of high velocity at the edge of the LCE, as well as the alignment of the layers with the geostrophic streamfunction also support this hypothesis. 


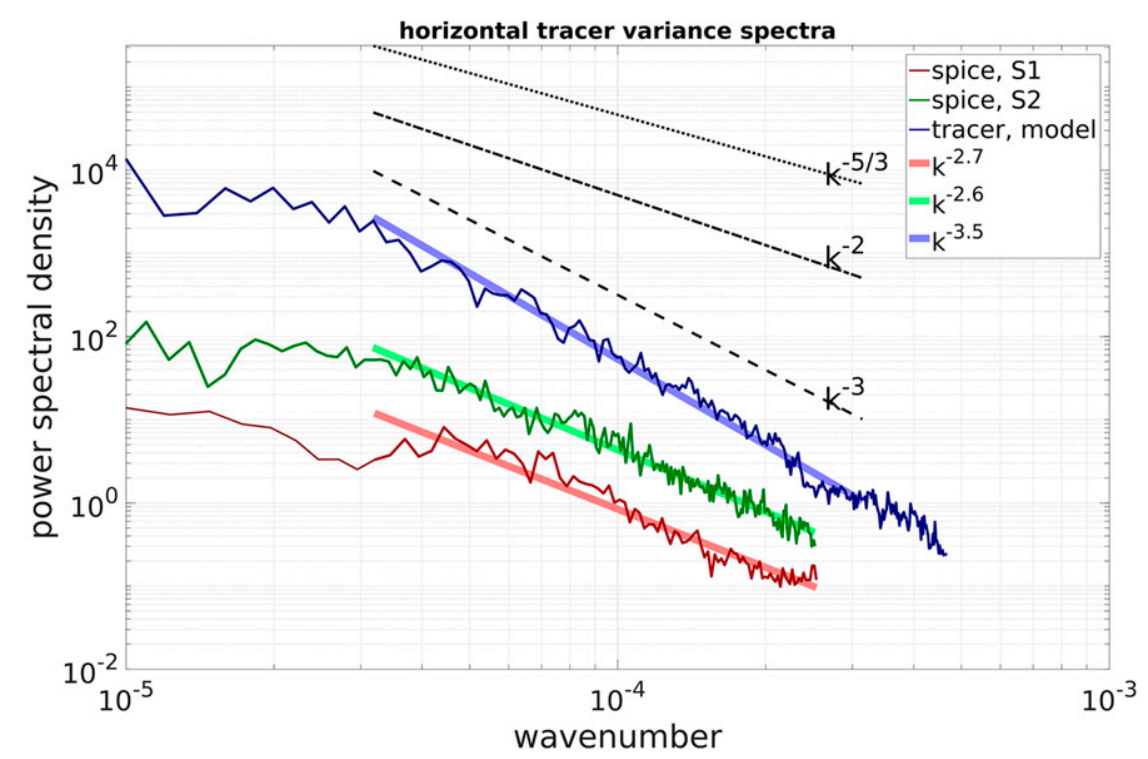

FIG. 11. Tracer variance spectra. The dark-green and dark-red lines are the spice variance spectra computed from glider sections 1 and 2, respectively. The dark-blue line is the modeled passive tracer variance spectra. The light-green, light-red, and light-blue thick lines represent the linear fit of the spectra in the $10^{-4.5}<k_{h}<10^{-3.5} \mathrm{~m}^{-1}$ wavenumber range (wavelengths of $3-30 \mathrm{~km}$ ). The $k^{-5 / 3}, k^{-2}$, and $k^{-3}$ slopes are indicated as black dotted lines.

The simulations from the QG model are consistent with these observations. Layering in the model first develops near the edges of the vortex, where the vertical shear of azimuthal velocity is strong, similar to the layer formation around interior lens-shaped vortices documented by Hua et al. (2013) and Meunier et al. (2015). The increased vertical scales of the modeled layers, compared to the observed layers, could be related to coarse model resolution $(5.3 \mathrm{~m})$ but ageostrophic and submesoscale effects, not incorporated into the model, could also play a role [e.g., symmetric instability (Brannigan et al. 2017) or near-inertial waves (Joyce et al. 2013)]. In particular, these latter processes can be associated with high vertical shear and, because they are not captured by the QG model, might account for these differences.

Results from the passive tracer advection model additionally help depict how layering patterns might emerge. Initialized with a velocity field consistent with a baroclinic surface-intensified anticyclonic vortex, the model begins to reveal layers forming in the areas of high vertical shear and that align with the streamfunction after only a few days of model integration. In particular, the initially barotropic tracer columns are tilted and deformed by the vertical shear of the azimuthal flow $u_{\phi}$. This process is the vertical equivalent of horizontal tracer stirring around a barotropic vortex (Rhines and Young 1983), a process illustrated schematically in Fig. 12. The layers reduce in thickness with increasing time, with their thickness scaling as

$$
\delta=L_{o} /\left(\partial_{z} u_{\phi} t\right)
$$

where $L_{o}$ is the initial diameter of the tracer column, $\partial_{z} u_{\phi}$ is the vertical shear of the flow, and $t$ is time. Meunier et al. (2015) further showed that the evolution of the tracer's vertical wavenumber $k_{z}=\partial_{z} \chi / \chi$ is driven completely by 1$)$ the vertical shear of azimuthal velocity $\partial_{z} u_{\phi}$ and 2) the initial azimuthal wavenumber, $k_{\phi}=\partial_{\phi} \chi /(r \chi)$. That is,

$$
d k_{z} / d t=-\partial_{z} u_{\phi} k_{\phi},
$$

which is a specific case of the direct vertical-scale cascade of tracers under the influence of shear and strain described by Haynes and Anglade (1997). Equation (14) demonstrates that 1) the vertical wavenumber increases with time (the vertical scale decreases) as azimuthal variability coincides with strong shear and 2) high vertical wavenumbers emerge faster when high azimuthal wavenumbers are present (i.e., small-scale azimuthal variations).

The steep spectral slopes of the modeled passive tracer shown in Fig. $11\left(\approx k_{h}^{-11 / 3}\right)$ are consistent with the theoretical and numerical results of Gilbert (1988) on 


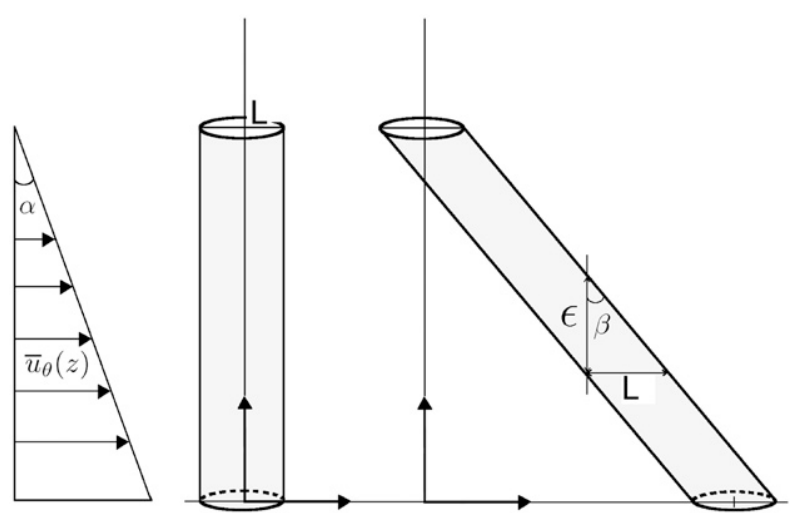

FIG. 12. Schematic view from the action of a vertically sheared flow $u_{\phi}(z)=u_{o}+\alpha z$ on a tracer tube of initial radius $L$, from Meunier et al. (2015). The tracer tube is tilted to an angle $\beta$, which depends on $\alpha$ and time.

the spectral slope of a passive tracer spiraling around a coherent vortex. The slightly flatter spectral slope found in both observed spice sections $\left(\approx k^{-2.7}\right)$, showing more energy at the high end of the spectrum, may suggest the importance of submesoscale flows in the LCE's spice distribution. Note, however, that the slope of spice spectra from sections 1 and 2 is steeper than the expected $k^{-2}$ slope for QG turbulence (Ferrari and Polzin 2005), supporting the idea of stirring of spice anomalies around a compact, coherent vortex à la Gilbert (1988).

In the nonlinear QG model, the same process occurs. Baroclinic instability generates tracer anomalies (whether PV or $\rho_{q}$ ). Although PV is an active tracer, it is materially conserved and is also subject to vertically differential advection. If the anomalies' amplitudes and length scales are sufficiently small relative to that of the mean flow, the anomalies behave like a passive tracer (Haynes 2001; Smith and Ferrari 2009). Baroclinic instability breaks the vortex's azimuthal symmetry as PV anomalies grow. As their contours cross the stream lines, the azimuthal advection term becomes nonzero and azimuthal variability is then transformed into vertical variability by the vertical shear of azimuthal velocity. Note that, in our QG model, the azimuthal variability is intrinsic because it is provided by the growth of unstable modes. However, any external source of azimuthal variability could trigger the same vertical cascade, including the interaction or merger of a stable LCE with neighboring submesoscale structures. These findings are consistent with those of Schmitt et al. (1986), who observed thermohaline intrusions immediately below the crown of high velocity of a Gulf Stream ring and suggested that the mesoscale vertical shear could trigger the formation of these intrusions.
As is usually the case when sampling large eddies with relatively slow vehicles such as gliders, the question of synopticity must be addressed. We note that the glider traverses the diameter of the LCE in 1 month, taking approximately 10 days to travel the maximum length scale over which coherent layering was observed $(120 \mathrm{~km})$. Simulations using the QG model suggest that layering is a persistent phenomenon: layering begins to form after about 10 days of integration and remains until the end of the simulations (Fig. 9d). However, the fate of each layer is more difficult to assess. From theoretical considerations and model results, we understand that during the eddy's lifetime, new layers form when azimuthal variability is provided, whether intrinsically or externally. They then reduce in thickness until they reach the dissipative scale, which arrests the cascade. In the case of a passive tracer, it is, however, possible to estimate the thinning rate of the layers using Eq. (13), which yields a time scale for layer formation (Meunier et al. 2015):

$$
\tau=L_{o} /\left(\partial_{z} u_{\phi} \delta\right)
$$

To better understand what is resolved by the glider, it is worth considering a couple cases in the evaluation of Eq. (15). Consider the case in which the azimuthal perturbation is provided by low-azimuthal-wavenumber baroclinic instability (say $m=2$ as in the QG simulation). Here, its azimuthal length scale is $L_{o} \approx \pi D /(2 m)$, where $D$ is the diameter of the eddy. Given an eddy diameter of $300 \mathrm{~km}$, corresponding to an azimuthal perturbation's length scale of $L_{o}=230 \mathrm{~km}$, the formation time scale of a $\delta=30$-m-thick layer by the vertical shear of the LCE Poseidon (i.e., $\partial_{z} u_{\phi}=5 \times 10^{-3} \mathrm{~s}^{-1}$ ) is therefore $\tau \approx 18$ days. Another 18 days later, the thickness of this same layer will be $15 \mathrm{~m}$. For a smaller perturbation scale, say having horizontal and vertical extents equal to $L_{o}=30 \mathrm{~km}$ and $\delta=30 \mathrm{~m}$, respectively, the formation time scale is about 2.3 days. Layers with these characteristics might arise, for example, through high-azimuthal-mode barotropic instability or preexisting submesoscale structures, and it would take only 1 month for the latter to reach the vertical sampling resolution of the glider $(2 \mathrm{~m})$. Of course, these are only approximate scaling arguments, and in the real ocean turbulent diffusion and double diffusion are expected to kick in and may arrest the cascade before the layers reach the meter scale. Nevertheless, these scales gives us useful information with which to compare that of the glider survey. It essentially shows that layering arising from mesoscale azimuthal perturbations (low-azimuthal unstable modes) can be considered as persistent and coherent over the glider survey's time scale [i.e. $O(1)$ 
month], whereas layering arising from submesoscale perturbations (high-azimuthal unstable modes or small neighboring eddies) is likely a more transient phenomenon; its lateral structure may be poorly resolved by the glider measurements.

In the observations that are presented here, double diffusive processes are likely of little importance for the generation of layering. The vertical distribution of the Turner angle does not show major variations along the sections. The same general pattern is observed: salt-finger unstable below the eddy, and convective-diffusion unstable in the core of the eddy. Although layering is discernible in the Turner angle sections, it is mostly associated with stabilization or weak convective-diffusion instability in an environment otherwise unstable to salt fingering. If double diffusion were responsible for the layering generation, the latter should distribute homogeneously below the eddy core, which is not the case here. Note, however, that double diffusive instabilities might play a major role in the diffusion of these layers once they reach a sufficiently small scale, as shown by Schmitt et al. (1986) in a Gulf Stream ring and numerous studies in meddies (Ruddick and Hebert 1988; Ruddick 1992).

Of particular interest is the fate of these layers, and their impact on the diffusion of the thermohaline content of the LCE. Layering was shown to be the leading-order process for the diffusion of heat and salt in the decay of meddies (Armi et al. 1989; Ruddick and Hebert 1988; Tokos and Rossby 1991). Moreover, a strong correlation exists between similar thermohaline fine structures and enhanced turbulent diffusion in Gulf Stream warm-core rings (Schmitt et al. 1986). In the absence of a possible quantification of the layeringmediated mixing under LCEs, however, one must remain cautious: while meddies are almost isolated structures drifting in a calm subthermocline environment so that establishing a direct link between layering and mixing is within reach, making such links for the case of LCEs might be a more challenging prospect. Warm-core rings are, in general, near-surface ventilated eddies (Dewar 1987, 1988), and atmospheric forcing in the Gulf of Mexico is particularly intense, including hurricanes and cold fronts. These latter processes result in pronounced momentum and heat fluxes that mix and cool the ocean as well as generate inertia gravity waves (IGWs) that penetrate deep into the ocean interior (D'Asaro 2014; Alford et al. 2016; Pallàs-Sanz et al. 2016). A detailed assessment of the erosion of LCE's heat and salt reservoir in the GoM's interior (i.e., distant from topographic boundaries) therefore involves disentangling the impacts of 1) layering, 2) wind- and buoyancy-driven turbulent mixing, and 3) breaking IGWs, among other dynamics. Decoupling the turbulence generated by such processes in both the upper ocean and interior ocean is an active area of research.

Atmospheric forcing was shown to be important and able to deepen the LCE's mixed layer down to $150 \mathrm{~m}$ (Muller-Karger et al. 2015; Damien et al. 2018), likely playing an important role in eroding the upper part of the LCE's temperature maximum. However, this winter mixed layer is still not deep enough to explain the saline core erosion seen here, because the latter resides between 200 and $600 \mathrm{~m}$. This suggests that, at least at these depths, both layering and IGWs might be the leading mechanisms. Both were shown to be important in the erosion of Gulf Stream rings. For example, Schmitt et al. (1986) showed that mixing was enhanced in the intrusion zones below the highvelocity annulus, consistent with earlier observations by Ruddick and Bennett (1985). Similarly, Lueck and Osborn (1986) found elevated turbulent kinetic energy dissipation near the edge of the ring but also showed that dissipation in the eddy's thermocline was driven by near-inertial wave trapping, independent of the large-scale vertical shear.

The similarity in observed structure between LCEs and Gulf Stream rings suggest that similar competing processes may occur below LCEs and encourages further observational and modeling efforts. Although our study reveals the existence of layering below LCEs and we propose a simple formation mechanism, an estimate of its impact on turbulence and mixing remains unexplored. Forthcoming glider surveys including direct microstructure measurements in LCEs could help to quantify the rate of turbulent diffusion, its spatial distribution, and a direct connection to layering. The use of more sophisticated primitive equation models, including wind forcing and surface heat fluxes and perhaps at high temporal and spatial resolution, also appears necessary to assess the contributions of each mechanism on LCE heat and salt loss.

An important conclusion resulting from our study is that a reflector's slope is not an accurate proxy of the isopycnal slopes for large-scale coherent reflectors (Krahmann et al. 2009). As did Ruddick (2018), it therefore questions the validity of using seismic reflectors to infer isopycnal slopes in zones of intrusions or interleaving. Figure 13 compares the slopes of the isopycnal and the geostrophic streamfunction with that of the reflectors in the southern half of glider section 1: the reflectors below the LCE align with the geostrophic streamfunction, whereas the isopycnals have more moderate slopes. The reflector's slopes are 


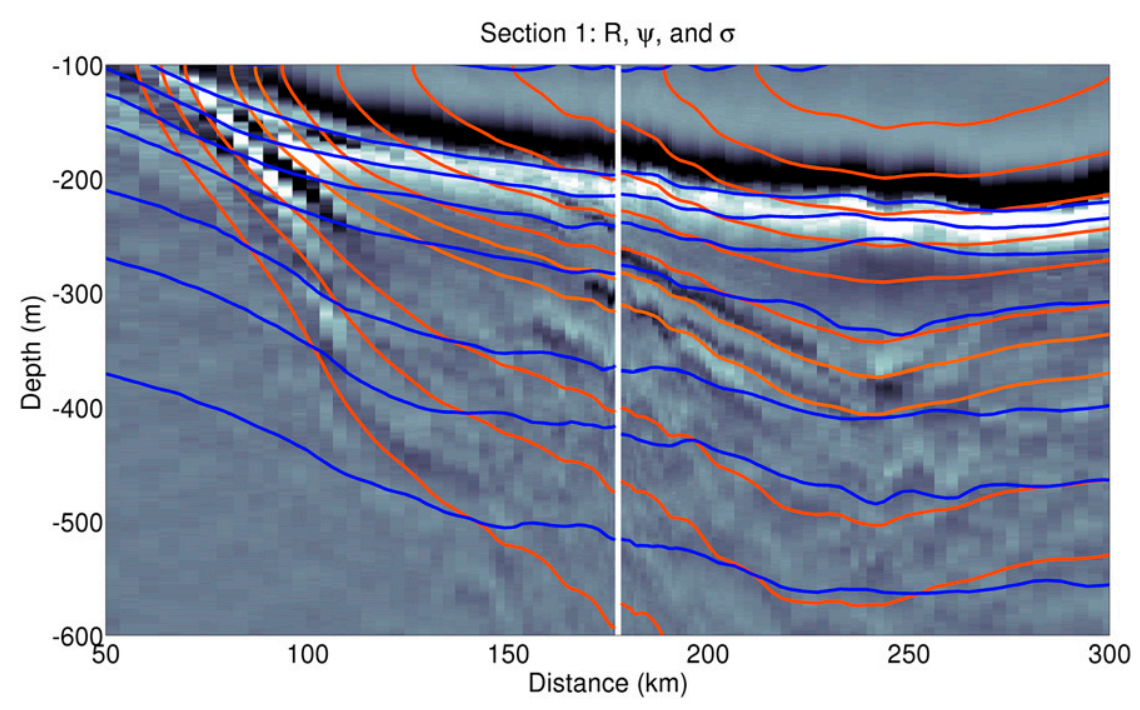

FIG. 13. Potential density (blue) and geostrophic streamfunction (orange) contours superimposed on a high-pass-filtered temperature vertical section (grayscale image).

thus a measure of the slope of the vertical integral of the density anomaly, rather than the density itself. This result suggests that one should be cautious when interpreting seismic images, particularly when trying to infer isopycnal slopes.

Acknowledgments. This research was funded by the National Council of Science and Technology of MexicoMexican Ministry of Energy-Hydrocarbon Trust, project 201441. This is a contribution of the Gulf of Mexico Research Consortium (CIGoM). The authors are grateful to Prof. Julio Sheinbaum and Dr. Sergei Molodstov for constructive discussions.

\section{REFERENCES}

Alford, M. H., J. A. MacKinnon, H. L. Simmons, and J. D. Nash, 2016: Near-inertial internal gravity waves in the ocean. Annu. Rev. Mar. Sci., 8, 95-123, https://doi.org/10.1146/annurevmarine-010814-015746.

Armi, L., D. Hebert, N. Oakey, J. F. Price, P. L. Richardson, H. Thomas Rossby, and B. Ruddick, 1989: Two years in the life of a Mediterranean salt lens. J. Phys. Oceanogr., 19, 354-370, https://doi.org/10.1175/1520-0485(1989)019<0354: TYITLO $>2.0 . \mathrm{CO} ; 2$.

Austin, G. B., 1955: Some recent oceanographic surveys of the Gulf of Mexico. Eos, Trans. Amer. Geophys. Union, 36, 885-892, https://doi.org/10.1029/TR036i005p00885.

Biescas, B., V. Sallarès, J. L. Pelegrí, F. Machín, R. Carbonell, G. Buffett, J. J. Dañobeitia, and A. Calahorrano, 2008: Imaging meddy finestructure using multichannel seismic reflection data. Geophys. Res. Lett., 35, L11609, https://doi.org/ 10.1029/2008GL033971.

Biggs, D. C., and F. E. Müller-Karger, 1994: Ship and satellite observations of chlorophyll stocks in interacting cycloneanticyclone eddy pairs in the western Gulf of Mexico.
J. Geophys. Res., 99, 7371-7384, https://doi.org/10.1029/ 93JC02153.

Brannigan, L., D. P. Marshall, A. C. Naveira Garabato, A. J. G. Nurser, and J. Kaiser, 2017: Submesoscale instabilities in mesoscale eddies. J. Phys. Oceanogr., 47, 3061-3085, https:// doi.org/10.1175/JPO-D-16-0178.1.

Cooper, C., G. Z. Forristall, and T. M. Joyce, 1990: Velocity and hydrographic structure of two Gulf of Mexico warm-core rings. J. Geophys. Res., 95, 1663-1679, https://doi.org/10.1029/ JC095iC02p01663.

D'Asaro, E. A., 2014: Turbulence in the upper-ocean mixed layer. Annu. Rev. Mar. Sci., 6, 101-115, https://doi.org/10.1146/ annurev-marine-010213-135138.

Damien, P., O. Pasqueron de Fommervault, J. Sheinbaum, J. Jouanno, V. F. Camacho-Ibar, and O. Duteil, 2018: Partitioning of the open waters of the Gulf of Mexico based on the seasonal and interannual variability of chlorophyll concentration. J. Geophys. Res. Oceans, https://doi.org/10.1002/ 2017JC013456.

Dewar, W. K., 1987: Ventilating warm rings: Theory and energetics. J. Phys. Oceanogr., 17, 2219-2231, https://doi.org/10.1175/ 1520-0485(1987)017<2219:VWRTAE > 2.0.CO;2.

_ 1988: Ventilating warm rings: Structure and model evaluation. J. Phys. Oceanogr., 18, 552-564, https://doi.org/10.1175/ 1520-0485(1988)018<0552:VWRSAM > 2.0.CO;2.

Dickinson, A., N. J. White, and C.-P. Caulfield, 2017: Spatial variation of diapycnal diffusivity estimated from seismic imaging of internal wave field, Gulf of Mexico. J. Geophys. Res. Oceans, 122, 9827-9854, https://doi.org/10.1002/ 2017JC013352.

Elliott, B. A., 1982: Anticyclonic rings in the Gulf of Mexico. J. Phys. Oceanogr., 12, 1292-1309, https://doi.org/10.1175/ 1520-0485(1982)012<1292:ARITGO > 2.0.CO;2.

Ferrari, R., and K. L. Polzin, 2005: Finescale structure of the T S Relation in the Eastern North Atlantic. J. Phys. Oceanogr., 35, 1437, https://doi.org/10.1175/JPO2763.1.

Flament, P., 2002: A state variable for characterizing water masses and their diffusive stability: spiciness. Prog. Oceanogr., 54, 493-501, https://doi.org/10.1016/S0079-6611(02)00065-4. 
Forristall, G. Z., K. J. Schaudt, and C. K. Cooper, 1992: Evolution and kinematics of a loop current eddy in the Gulf of Mexico during 1985. J. Geophys. Res., 97, 2173-2184, https://doi.org/ 10.1029/91JC02905.

Garau, B., S. Ruiz, W. G. Zhang, A. Pascual, E. Heslop, J. Kerfoot, and J. Tintoré, 2011: Thermal lag correction on slocum CTD glider data. J. Atmos. Oceanic Technol., 28, 1065-1071, https:// doi.org/10.1175/JTECH-D-10-05030.1.

Gilbert, A. D., 1988: Spiral structures and spectra in two-dimensional turbulence. J. Fluid Mech., 193, 475-497, https://doi.org/10.1017/ S0022112088002228.

Hamilton, P., R. Leben, A. Bower, H. Furey, and P. Pérez-Brunius, 2018: Hydrography of the Gulf of Mexico using autonomous floats. J. Phys. Oceanogr., 48, 773-794, https://doi.org/10.1175/ JPO-D-17-0205.1.

Haynes, P. H., 2001: Vertical shear plus horizontal stretching as a route to mixing. From Stirring to Mixing in a Stratified Ocean: Proc. 12th 'Aha Huliko'a Hawaiian Winter Workshop, Honolulu, HI, University of Hawai'i at Mānoa, 73-79, http:// www.soest.hawaii.edu/PubServices/2001pdfs/Haynes.pdf.

__ , and J. Anglade, 1997: The vertical-scale cascade in atmospheric tracers due to large-scale differential advection. J. Atmos. Sci., 54, 1121-1136, https://doi.org/10.1175/ 1520-0469(1997)054<1121:TVSCIA > 2.0.CO;2.

Hernández-Guerra, A., and T. M. Joyce, 2000: Water masses and circulation in the surface layers of the Caribbean at $66^{\circ} \mathrm{W}$. Geophys. Res. Lett., 27, 3497-3500, https://doi.org/10.1029/1999GL011230.

Hua, B. L., and D. B. Haidvogel, 1986: Numerical simulations of the vertical structure of quasi-geostrophic turbulence. J. Atmos. Sci., 43, 2923-2936, https://doi.org/10.1175/1520-0469(1986) 043<2923:NSOTVS $>2.0$. CO 2 .

—, C. Ménesguen, S. Le Gentil, R. Schopp, B. Marsset, and H. Aiki, 2013: Layering and turbulence surrounding an anticyclonic oceanic vortex: In situ observations and quasi-geostrophic numerical simulations. J. Fluid Mech., 731, 418-442, https:// doi.org/10.1017/jfm.2013.369.

Joyce, T. M., J. M. Toole, P. Klein, and L. N. Thomas, 2013: A nearinertial mode observed within a Gulf Stream warm-core ring. J. Geophys. Res. Oceans, 118, 1797-1806, https://doi.org/ 10.1002/jgrc.20141.

Krahmann, G., C. Papenberg, P. Brandt, and M. Vogt, 2009: Evaluation of seismic reflector slopes with a Yoyo-CTD. Geophys. Res. Lett., 36, L00D02, https://doi.org/10.1029/2009GL038964.

Leben, R. R., 2005: Altimeter-derived loop current metrics. Circulation in the Gulf of Mexico: Observations and Models, Geophys. Monogr., Vol. 161, Amer. Geophys. Union, 181-201, https:// doi.org/10.1029/161GM15.

Ledwell, J. R., R. He, Z. Xue, S. F. DiMarco, L. J. Spencer, and P. Chapman, 2016: Dispersion of a tracer in the deep Gulf of Mexico. J. Geophys. Res. Oceans, 121, 1110-1132, https:// doi.org/10.1002/2015JC011405.

Leipper, D. F., 1970: A sequence of current patterns in the Gulf of Mexico. J. Geophys. Res., 75, 637-657, https://doi.org/10.1029/ JC075i003p00637.

Lipphardt, B., A. Poje, A. Kirwan, L. Kantha, and M. Zweng, 2008: Death of three loop current rings. J. Mar. Res., 66, 25-60, https://doi.org/10.1357/002224008784815748.

Lueck, R. G., 1990: Thermal inertia of conductivity cells: Theory. J. Atmos. Oceanic Technol., 7, 741-755, https://doi.org/ 10.1175/1520-0426(1990)007<0741:TIOCCT>2.0.CO;2.

— , and T. Osborn, 1986: The dissipation of kinetic energy in a warm-core ring. J. Geophys. Res., 91, 803-818, https://doi.org/ 10.1029/JC091iC01p00803.
Ménesguen, C., B. L. Hua, X. Carton, F. Klingelhoefer, P. Schnürle, and C. Reichert, 2012: Arms winding around a meddy seen in seismic reflection data close to the Morocco coastline. Geophys. Res. Lett., 39, L05604, https://doi.org/10.1029/2011GL050798.

, S. Le Gentil, P. Marchesiello, and N. Ducousso, 2018: Destabilization of an oceanic meddy-like vortex: Energy transfers and significance of numerical settings. J. Phys. Oceanogr., 48, 1151-1168, https://doi.org/10.1175/jpo-d-17-0126.1.

Meunier, T., C. Ménesguen, R. Schopp, and S. Le Gentil, 2015: Tracer stirring around a meddy: The formation of layering. J. Phys. Oceanogr., 45, 407-423, https://doi.org/10.1175/ JPO-D-14-0061.1.

, - - X. Carton, S. Le Gentil, and R. Schopp, 2018a: Optimal perturbations of an oceanic vortex lens. Fluids, 3, 63, https:// doi.org/10.3390/fluids3030063.

— , E. Pallás-Sanz, M. Tenreiro, E. Portela, J. Ochoa, A. RuizAngulo, and S. Cusí, 2018b: The vertical structure of a loop current eddy. J. Geophys. Res. Oceans, 123, 6070-6090, https:// doi.org/10.1029/2018JC013801.

— , and Coauthors, 2018c: Intrathermocline eddies embedded within an anticyclonic vortex ring. Geophys. Res. Lett., 45 , 7624-7633, https://doi.org/10.1029/2018GL077527.

Muller-Karger, F. E., and Coauthors, 2015: Natural variability of surface oceanographic conditions in the offshore Gulf of Mexico. Prog. Oceanogr., 134, 54-76, https://doi.org/10.1016/ j.pocean.2014.12.007.

Nguyen, H. Y., B. L. Hua, R. Schopp, and X. Carton, 2012: Slow quasigeostrophic unstable modes of a lens vortex in a continuously stratified flow. Geophys. Astrophys. Fluid Dyn., 106, 305-319, https://doi.org/10.1080/03091929.2011.620568.

Pallàs-Sanz, E., J. Candela, J. Sheinbaum, and J. Ochoa, 2016: Mooring observations of the near-inertial wave wake of Hurricane Ida (2009). Dyn. Atmos. Oceans, 76, 325-344, https://doi.org/10.1016/ j.dynatmoce.2016.05.003.

Pinheiro, L. M., H. Song, B. Ruddick, J. Dubert, I. Ambar, K. Mustafa, and R. Bezerra, 2010: Detailed 2-D imaging of the Mediterranean outflow and meddies off W Iberia from multichannel seismic data. J. Mar. Syst., 79, 89-100, https://doi.org/ 10.1016/j.jmarsys.2009.07.004.

Portela, E. B., M. Tenreiro, E. Pallas-Sanz, T. Meunier, A. RuizAngulo, E. Sosa-Gutierrez, and S. Cusi, 2018: Hydrography of the central-western Gulf of Mexico. J. Geophys. Res. Oceans, 123, 5134-5149, https://doi.org/10.1029/2018JC013813.

Rhines, P. B., and W. R. Young, 1983: How rapidly is a passive scalar mixed within closed streamlines? J. Fluid Mech., 133, 133-145, https://doi.org/10.1017/S0022112083001822.

Rice, A. E., J. W. Book, W. T. Wood, and T. Fischer, 2013: Currenteddy interaction in the Agulhas Return Current region from the seismic oceanography perspective. J. Acoust. Soc. Amer., 133, 3314, https://doi.org/10.1121/1.4805515.

Ruddick, B., 1983: A practical indicator of the stability of the water column to double-diffusive activity. Deep-Sea Res., 30A, 1105-1107, https://doi.org/10.1016/0198-0149(83)90063-8.

_ 1992: Intrusive mixing in a Mediterranean salt lens-Intrusion slopes and dynamical mechanisms. J. Phys. Oceanogr., 22, 1274-1285, https://doi.org/10.1175/1520-0485(1992)022<1274: IMIAMS $>2.0 . \mathrm{CO} ; 2$.

, 2018: Seismic oceanography's failure to flourish: A possible solution. J. Geophys. Res. Oceans, 123, 4-7, https://doi.org/ 10.1002/2017JC013736.

_ and A. S. Bennett, 1985: Fine structure and mixing at the edge of a warm core ring. J. Geophys. Res., 90, 8943-8951, https:// doi.org/10.1029/JC090iC05p08943. 
, and D. Hebert, 1988: The mixing of Meddy "Sharon." SmallScale Turbulence and Mixing in the Ocean: Proceedings of the 19th International Liege Colloquium on Ocean Hydrodynamics, J. C. J. Nihoul and B. M. Jamart, Eds., Elsevier Oceanography Series, Vol. 46, Elsevier, 249-261.

, and K. Richards, 2003: Oceanic thermohaline intrusions: observations. Prog. Oceanogr., 56, 499-527, https://doi.org/ 10.1016/S0079-6611(03)00028-4.

Rudnick, D. L., G. Gopalakrishnan, and B. D. Cornuelle, 2015: Cyclonic eddies in the Gulf of Mexico: Observations by underwater gliders and simulations by numerical model. J. Phys. Oceanogr., 45, 313-326, https://doi.org/10.1175/JPO-D-14-0138.1.

Sallarès, V., B. Biescas, G. Buffett, R. Carbonell, J. J. Dañobeitia, and J. L. Pelegrí, 2009: Relative contribution of temperature and salinity to ocean acoustic reflectivity. Geophys. Res. Lett., 36, L00D06, https://doi.org/10.1029/2009GL040187.

Schmitt, R. W., R. G. Lueck, and T. M. Joyce, 1986: Fine- and microstructure at the edge of a warm-core ring. Deep-Sea Res., 33A, 1665-1689, https://doi.org/10.1016/0198-0149(86) 90073-7.

Smith, K. S., and R. Ferrari, 2009: The production and dissipation of compensated thermohaline variance by mesoscale stirring. J. Phys. Oceanogr., 39, 2477, https://doi.org/10.1175/ 2009JPO4103.1.
Song, H., L. M. Pinheiro, B. Ruddick, and F. C. Teixeira, 2011: Meddy, spiral arms, and mixing mechanisms viewed by seismic imaging in the Tagus Abyssal Plain (SW Iberia). J. Mar. Res., 69, 827-842, https://doi.org/10.1357/002224011799849309.

Tokos, K. S., and T. Rossby, 1991: Kinematics and dynamics of a Mediterranean salt lens. J. Phys. Oceanogr., 21, 879-892, https:// doi.org/10.1175/1520-0485(1991)021<0879:KADOAM>2.0.CO;2.

Turner, J. S., 1980: Buoyancy Effects in Fluids. Cambridge University Press, $382 \mathrm{pp}$.

Vidal, V. M. V., F. V. Vidal, and J. M. Pérez-Molero, 1992: Collision of a loop current anticyclonic ring against the continental shelf slope of the western Gulf of Mexico. J. Geophys. Res., 97, 2155-2172, https://doi.org/10.1029/91JC00486.

Wüst, G., 1964: Stratification and Circulation in the AntilleanCaribbean Basins. Columbia University Press, 201 pp.

Xu, F.-H., Y.-L. Chang, L.-Y. Oey, and P. Hamilton, 2013: Loop current growth and eddy shedding using models and observations: Analyses of the July 2011 eddy-shedding event. J. Phys. Oceanogr., 43, 1015-1027, https://doi.org/10.1175/ JPO-D-12-0138.1.

Yamashita, M., K. Yokota, Y. Fukao, S. Kodaira, S. Miura, and K. Katsumata, 2011: Seismic reflection imaging of a warm core ring south of Hokkaido. Explor. Geophys., 42, 18-24, https:// doi.org/10.1071/EG11004. 